\title{
Improvement of Anisotropy Sensitivity in the Scanning Acoustic Microscope
}

\author{
ABDULLAH ATALAR
}

\begin{abstract}
The response of the conventional scanning acoustic microscope (SAM) to anisotropic materials is theoretically investigated. For this purpose, the reflection coefficient of plane acoustic waves incident on a liquid-solid interface is numerically calculated for a general anisotropic solid oriented in any arbitrary direction. In general, the reflection coefficient depends on polar and azimuthal angles of incidence. For the case of a circularly symmetric acoustic microscope lens, a mean reflectance function can be defined that depends only on the polar angle. With this mean reflectance function it is very easy to predict the anisotropic material response of the acoustic microscope. Moreover, one can explore the effects of changing lens parameters such as the acoustic fields at the back side of the lens. It is found that under certain conditions, the amplitude response of the acoustic microscope can depend heavily on the orientation of the solid material under investigation. The amplitude of the acoustic microscope signal is influenced by the orientation of the material because there is a cancellation of acoustic rays reflected from the object surface at different azimuthal angles. This cancellation is revealed as a minimum in the mean reflectance function. It is shown by numerical simulation that, the sensitivity to orientation can be increased by use of a ring-shaped insonification at the back of the acoustic lens. With such lenses it may be possible to determine the orientation of crystallites in a material with a high lateral resolution.
\end{abstract}

\section{INTRODUCTION}

$\mathrm{T}$ HE scanning acoustic microscope (SAM) has become a useful new instrument for nondestructive testing applications by its ability to penetrate optically opaque materials [1]. It is possible to use it in characterizing the properties of materials on a microscope scale [2]. Crystalline materials such as integrated circuits and thin film structures are typically acoustically anisotropic. For such materials direction sensitive acoustic lens geometries have been utilized for characterization purposes: Kushibiki et al. [3]. [4] proposed and used successfully a cylindrically focused lens to get direction sensitive information with the complete loss of resolution in one direction. Hildebrand and Lam [5] produced a lens with separate transmitter and receiver to get a partially directive geometry with a slight loss in resolution. Tan et al. [6] reported a geometry containing two or three confocal lenses, again with a sensitivity to anistropy of the materials under investigation. Davids and Bertoni [7] proposed a special transducer geometry to increase the sensitivity to anisotropy without losing very much in the lateral resolu-

Manuscript received May 6. 1988.

The author is with the Electrical and Electronies Engineering Departmen1, P.K. 8 Maltepe. Ankara 06572, Turkey

IEEE Log Number 8824738 . tion. Khuri-Yakub and Chou [8], [9] used a shear wave transducer instead of the longitudinal transducer in the regular lens arrangement. The acoustic transmission characteristics of shear waves from the lens material to liquid generates a circularly asymmetrical field pattern that in turn produces a direction sensitive arrangement.

In all cases, the material dependent images as obtained from the acoustic microscope are a result of the sound wave reflection at the liquid-solid interface. The reflection coefficient-both amplitude and phase-at this interface uniquely characterizes the material under investigation. To understand the images and the information they convey, a thorough understanding of reflection coefficient is necessary.

Theoretical aspects of the reflection problem for the anisotropic solid case and some numerical calculations for special cases [10]-[12] can be found in the literature. Somekh et al. [13] have studied the reflection coefficient of anisotropic materials at a liquid interface for the purpose of understanding acoustic images obtained by acoustic microscopes. They have numerically calculated the reflection coefficients for some materials and applied the results for interpretation of contrast in acoustic images. Crean and Waintal [14] have calculated Rayleigh wave velocity on anisotropic substrates for the same purpose

In this paper, first, the results of a study on the reflection and of plane acoustical waves at a liquid-anisotropic-solid interface are presented. Then, the results are used to determine the response of the scanning acoustic microscope to anistropic materials. Finally, a way of improving the sensitivity to anisotropy is proposed.

\section{Response of the Acoustic Microscope to Anisotropic Materials}

For a planar object surface placed perpendicular to the lens axis, the scanning acoustic microscope output can be expressed in its nonparaxial form as [15]

$$
\begin{aligned}
V(Z)= & K \int_{-\infty}^{+\infty} \int_{-\infty}^{+\infty}\left[u^{+}(x, y) P(x, y)\right]^{2} R\left(\frac{x}{f}, \frac{y}{f}\right) \\
& \cdot \exp \left(-j \frac{4 \pi Z}{\lambda} \sqrt{1-\left(\frac{x}{f}\right)^{2}-\left(\frac{y}{f}\right)^{2}}\right) d x d y
\end{aligned}
$$

where $V$ is the output voltage of the acoustic microscope, $K$ is a proportionality constant, $u^{+}$is the acoustic field 
(scalar potential of the particle velocity field) at the back side of the acoustic lens, $P$ is the pupil function of the lens, $f$ is the focal length of the lens, $R$ is the complex reflectance function at the liquid-solid interface, $\lambda$ is the wavelength of acoustic waves in the liquid medium, and $Z$ is the distance between the focal point and the object position. This expression is valid for the most general case when the lens geometry and/or the reflectance function are not circularly symmetric. We point out that for anisotropic materials, $\mathbb{R}$ does not have circular symmetry. We also note that, the extra $\sqrt{1-(x / f)^{2}-(y / f)^{2}}$ factor in the denominator of the above integral as derived by Liang et al., [16] applies to a spherical transducer with no buffer medium. That factor cancels out with a cosine factor in the nominator for the conventional acoustic lens arrangement, so the equation above remains valid for nonparaxial cases as well. Equation (1) can be written in cylindrical coordinates as

$$
\begin{aligned}
V(Z)= & K \int_{0}^{2 \pi} \int_{0}^{r_{m}}\left[u^{+}(r \cos \phi, r \sin \phi)\right. \\
& \cdot P(r \cos \phi, r \sin \phi)]^{2} \\
& \cdot R\left(\frac{r}{f} \cos \phi, \frac{r}{f} \sin \phi\right) \exp [-j(4 \pi Z / \lambda) \\
& \left.\cdot \sqrt{1-(r / f)^{2}}\right] r d r d \phi
\end{aligned}
$$

where $r$ is radial distance, $\left(x^{2}+y^{2}\right)^{1 / 2}, \phi$ is the azimuthal angle, $\tan ^{-1}(y / x)$, and $r_{m}$ is the pupil radius. For a circularly symmetric lens geometry, the functions $u^{+}$and $P$ can be written as functions of $r$ only, but the $\phi$ dependence of $R$ remains. For the purposes of simplifying this integral, it is possible to define a mean reflectance function [13], $\mathbb{R}^{\prime}$, as

$$
R^{\prime}(\sin \theta)=\int_{0}^{2 \pi} R(\sin \theta \cos \phi, \sin \theta \sin \phi) d \phi
$$

where $\sin \theta=r / f$. Here, $R^{\prime}$ is a function of $\sin \theta$ only, since the $\phi$ dependence is removed by an integration in $\phi$ direction.

Using this definition for a circularly symmetric insonification and pupil function, (2) can be simplified. Combining (3) with (2) one can arrive at a one-dimensional integral

$$
\begin{aligned}
V(Z)= & K f^{2} \int_{0}^{\sin \theta_{m}} \sin \theta\left[u^{+}(f \sin \theta)\right. \\
& \cdot P(f \sin \theta)]^{2} R^{\prime}(\sin \theta) \\
& \cdot \exp \left(-j \frac{4 \pi Z}{\lambda} \sqrt{1-\left(\frac{r}{f}\right)^{2}}\right) d(\sin \theta)
\end{aligned}
$$

where $\sin \theta_{m}=r_{m} / f$.

The evaluation of this integral requires the determination of the mean reflectance function, $R^{\prime}$, and thus $R$. A formulation of the reflection coefficient problem to deter-
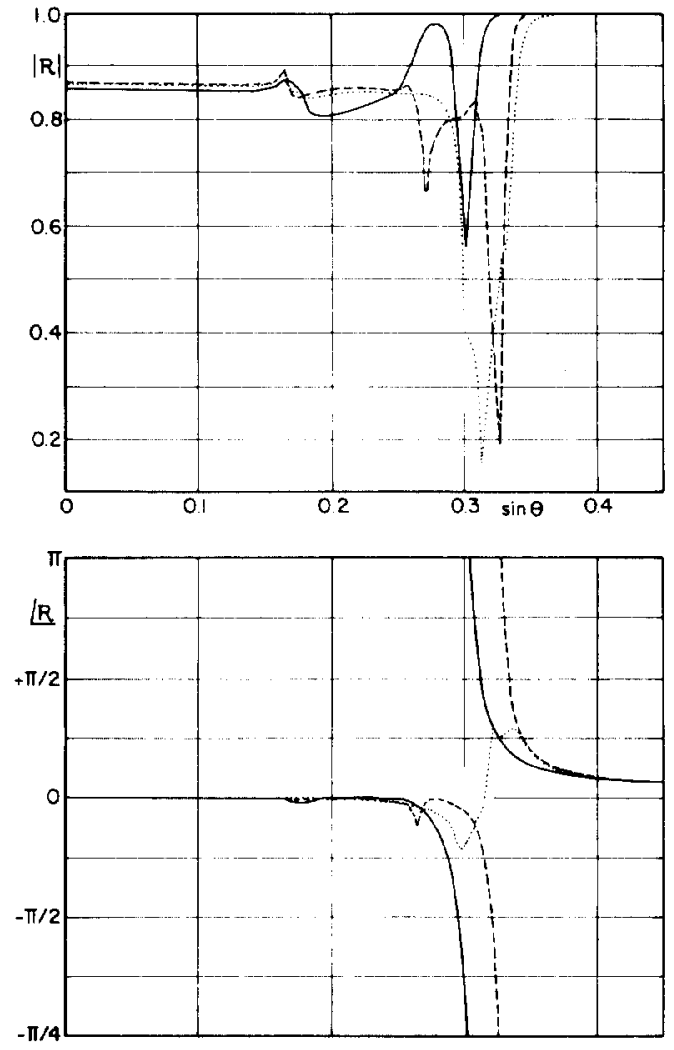

Fig. 1. Mean reflection function amplitude and phase for water-silicon in terfaces at various orientations as function of $\sin \theta$ for $(001),(011)$, and (111) surfaces shown by solid, dotted, and dashed lines, respectively.

mine $\mathbb{A}$ for the liquid-anisotropic-solid interface can be found elsewhere [17]. The formulation ignores acoustical attenuation and piezoelectricity of both media. The media are assumed to be of infinite extent and the interface is assumed to be an infinite plane. Due to the complexity of the expressions, an analytical solution is not possible, hence the solutions are obtained by numerical calculations. The developed computer program can handle materials with arbitrary stiffness matrices of 21 constants, so materials with arbitrary orientation can be handled with proper transformation of stiffness matrix by multiplication with Bond matrices [18]. The program is tested with some anisotropic materials whose reflection coefficients were previously calculated [12], [13].

The integral in (3) must be carefully evaluated because of the complex nature of the reflectance function. Sufficiently many points must be included in the integral for correct results, especially for large $\sin \theta$ values. We have calculated a number of such curves for the purpose of $V(Z)$ response determination. Fig. 1 shows the amplitude and phase plots of $R^{\prime}$ for water-silicon interface at principal planes of silicon. Since silicon crystal is of cubic structure, for (001) surface integration is performed for $\phi$ between $0^{\circ}$ and $45^{\circ}$, for (011) surface between $0^{\circ}$ and $90^{\circ}$ and for (111) surface between $0^{\circ}$ and $60^{\circ}$. Notice that, amplitude curves have an unusual structure near $\sin$ $\theta=0.3$. For (011) and (111) surfaces the mean reflection coefficient becomes as low as 0.2 . Moreover, the phase 

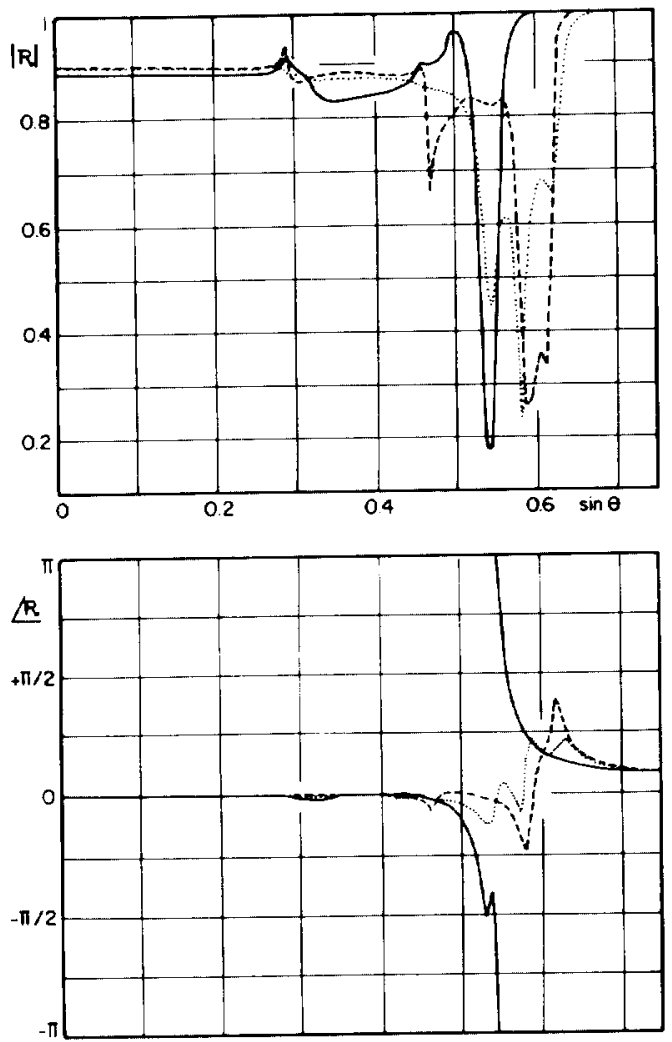

Fig. 2. Mean reflection function amplitude and phase for water-GaAs interface at various orientations as function of $\sin \theta$ for $(001),(011)$, and (111) surfaces shown by solid, dotted, and dashed lines, respectively.
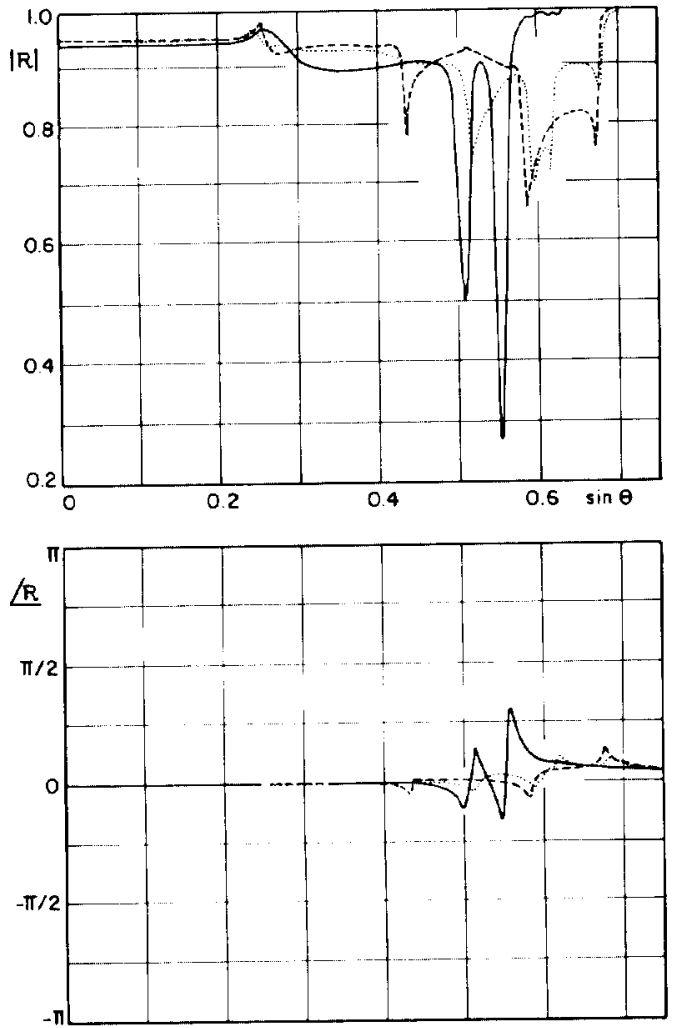

Fig. 3. Mean reflection function amplitude and phase for water-nickel interface at various orientations as function of $\sin \theta$ for $(001),(011)$, and (111) surfaces shown by solid, dotted, and dashed lines, respectively.
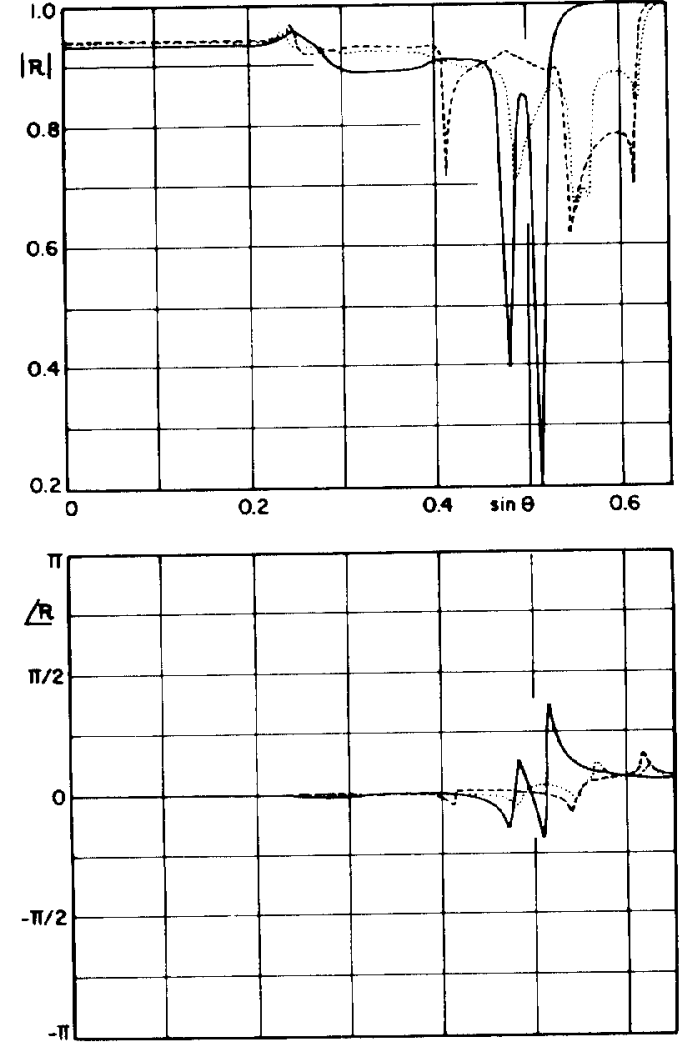

Fig. 4. Mean reflection function amplitude and phase for water-iron interface at various orientations as function of $\sin \theta$ for $(001),(011)$, and (111) surfaces shown by solid, dotted, and dashed lines, respectively.
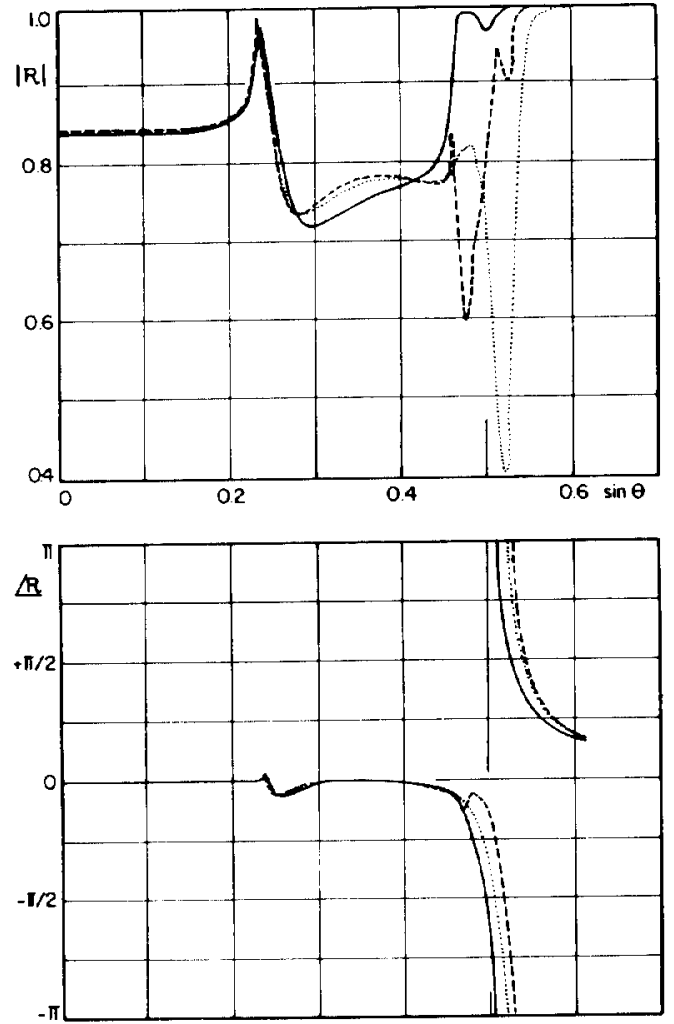

Fig. 5. Mean reflection function amplitude and phase for water-aluminum interface at various orientations as a function of $\sin \theta$ for $(001),(011)$, and (111) surface shown by solid, dotted, and dashed lines, respectively, 

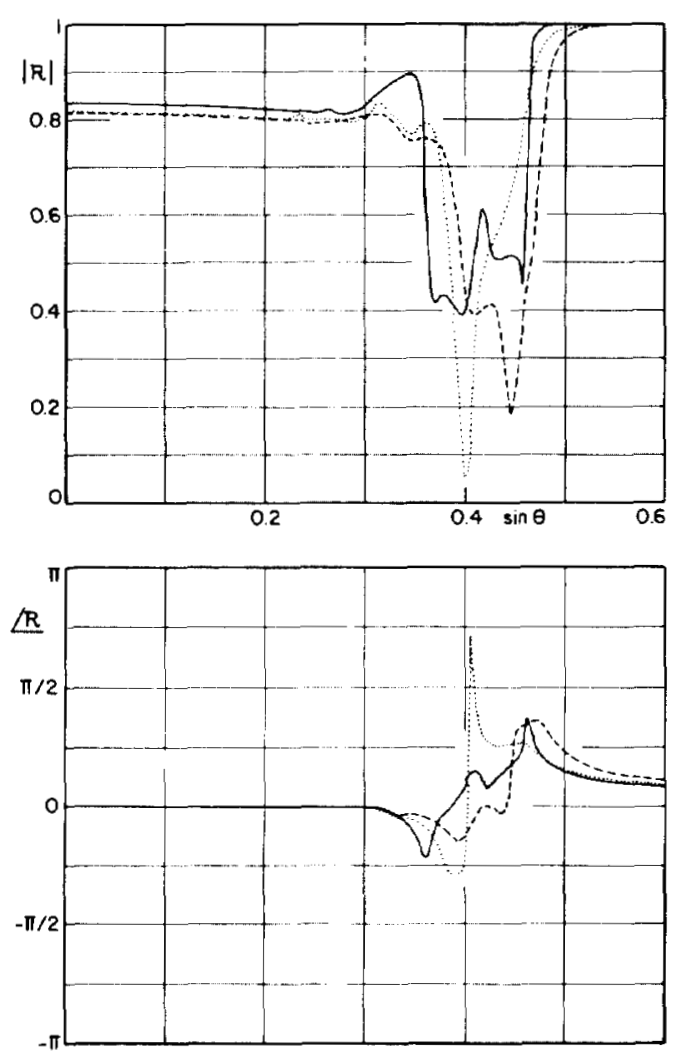

Fig. 6. Mean reflection function amplitude and phase for water-quartz interface at various orientations as a function of $\sin \theta$ for $(001),(100)$, and (010) surface shown by solid, dotted, and dashed lines, respectively.

curve for (011) surface does not have the usual $2 \pi$ phase transition. Figs. 2-5 are similar presentations for waterGaAs, -nickel, -iron, and -aluminum interfaces. Figs. 6 and 7 depict $R^{\prime}$ for two trigonal crystals, quartz and sapphire, also at the water interface for their $(100),(010)$ and (001) surfaces. For these calculations the integration ranges for $\phi$ at different orientations are as follows: (001) surface between $0^{\circ}$ and $30^{\circ},(100)$ surface between $0^{\circ}$ and $90^{\circ}$ and $(010)$ surface between $0^{\circ}$ and $180^{\circ}$. To test the validity of our computer program, we verified that the relation $R^{\prime}=\mathbb{R}$ holds true for isotropic solids. For anisotropic solids the $\phi$ step size is made successively smaller until a no significant change in $R^{\prime}$ is observed. In all cases, a $\sin \theta$ step size of 0.0015 , and a $\phi$ step size of $0.5^{\circ}$ are used. Elastic constants of solids were taken from Auld [18]. The constants for water are taken as follows: $c_{11}=2.277 \times 10^{9}$ and $\rho=1.0 \times 10^{3}$ in MKS units.

After inspection of these plots we make the following observations: The longitudinal wave critical angle for which the reflections coefficient amplitude reaches one, seems to disappear. Most of the mean reflectance functions have significant dips in amplitude and in some cases the 2- $\pi$ phase transition disappears. The dips in the curves occur near the phase transition and they are as a result of cancellation of reflected rays at different azimuthal angles after the integration operation. The cancellation is insignificant for isotropic or nearly isotropic materials where the phase transition occurs nearly at the same $\theta$ for different $\phi$ values. Recall that, the angular position of the phase
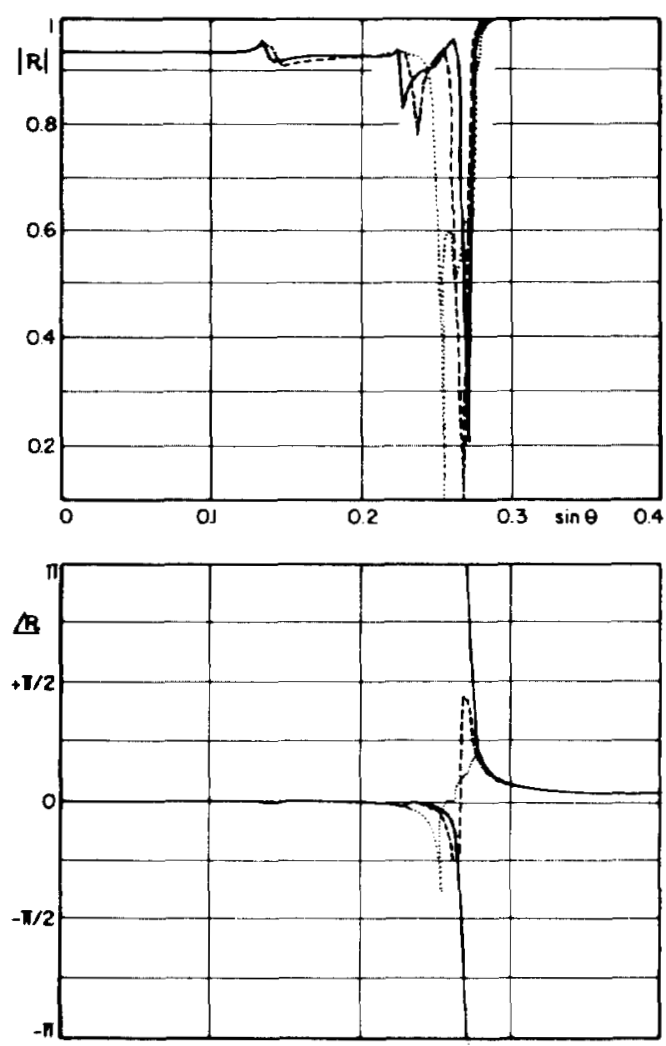

Fig. 7. Mean reflection function amplitude and phase for water-sapphire interface at various orientations as a function of $\sin \theta$ for $(001),(100)$, and $(010)$ surface shown by solid. dotted, and dashed lines, respectively.

transition is related to the Rayleigh wave velocity, and that for isotropic materials Rayleigh wave velocity is the same for all directions. But for anisotropic materials the phase transition occurs at different $\theta$ values for different $\phi$ values, because the Rayleigh wave velocity varies as a function of direction. When the acoustic rays at these angles are vectorially added, there is a cancellation. We must point out that our calculated curves for $R^{\prime}$ do not agree with Figs. 8-10 of Somekh et al. [13] although the curves for $R$ are in perfect agreement with their Fig. 4 . The discrepancy between the results may be explained by insufficient sampling in their integration computations [19].

We recall that the interference effect, which gives rise to material dependence of $V(Z)$ curves, occurs between the central rays and the rays near the phase transition angle [20]. For most anisotropic materials we observe that the amplitude of the rays near transition angle is considerably reduced because of the cancellation effect mentioned above. For a number of cases, the $2-\pi$ phase transition does not exist at all. Hence, the nulls and peaks of $V(Z)$ will not be very deep and for such materials the sensitivity to material parameters will be small.

Once $R^{\prime}$ is found, the integral of (4) is relatively easy to calculate. The functions $u^{+}$and $P$ are determined from the lens parameters. Typically one uses transducer sizes which will minimize the diffraction loss in the buffer rod to maximize the signal-to-noise ratio (SNR) of the acoustic microscope system. As an example we consider a lens with cavity radius of $105 \mu \mathrm{m}$, a pupil radius of $75 \mu \mathrm{m}$, 


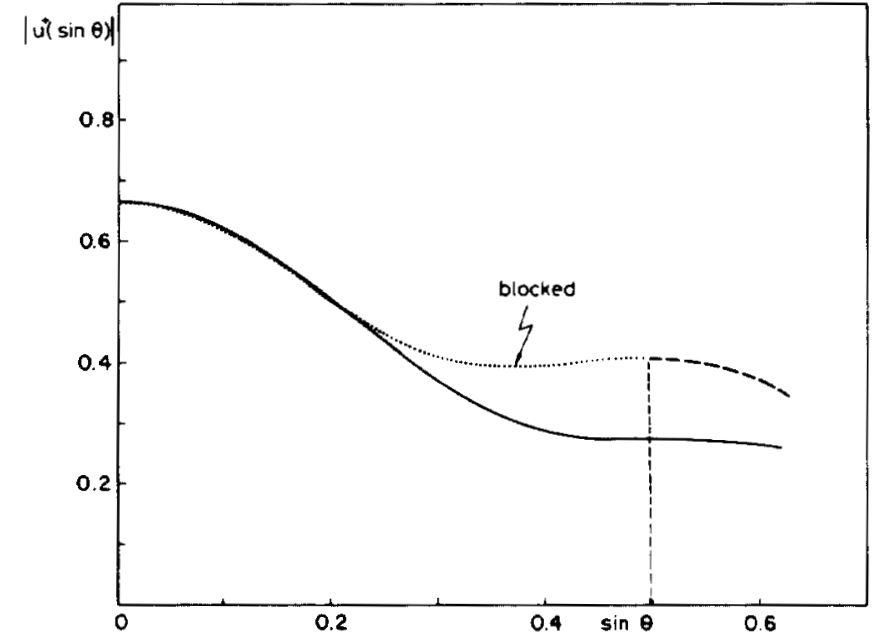

Fig. 8. Insonification functions used in computations. Solid curve: designed to minimize diffraction loss $\left(a_{1}=100 \mu \mathrm{m}, a_{2}=75 \mu \mathrm{m}, d=\right.$ $1000 \mu \mathrm{m}, \lambda=10.1 \mu \mathrm{m})$. Dashed curve: designed to improve sensitivity to anisotropy $\left(a_{1}=120 \mu \mathrm{m}, a_{2}=75 \mu \mathrm{m}, b_{2}=60 \mu \mathrm{m}, d=1140 \mu \mathrm{m}\right.$ $\lambda=10.1 \mu \mathrm{m})$. Dotted curve: blocked part.

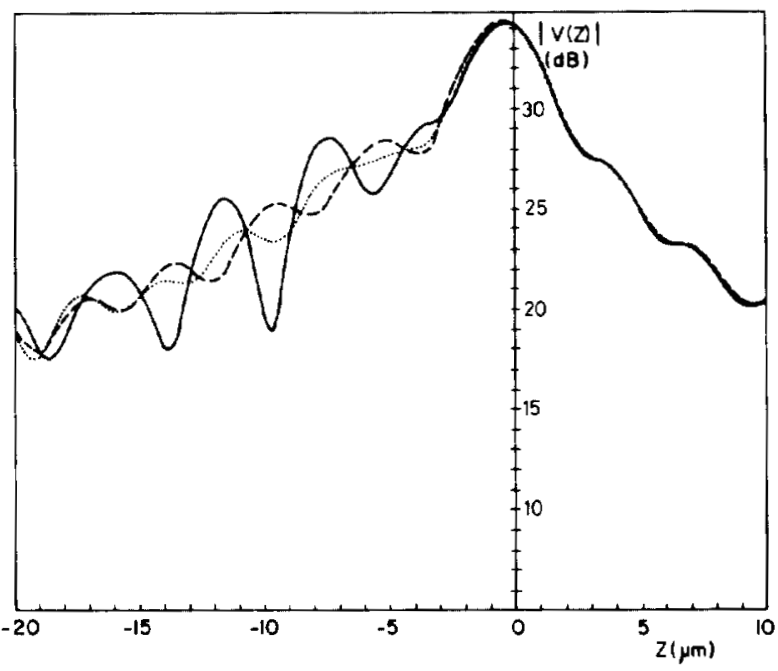

Fig. 9. Calculated $V(Z)$ curves for $(001)$, (011), and (111) faces of gallium arsenide, shown by solid. dotted, and dashed lines, respectively.

placed at a sapphire buffer rod length of $1000 \mu \mathrm{m}$ and used at a frequency of $1100 \mathrm{MHz}$. The optimum transducer radius turns out to be $100 \mu \mathrm{m}$. The solid curve in Fig. 8 shows the plot of $\left|u^{+}\right|$for this case. The pupil function, $P$, includes the effect of the antireflection layer on the lens surface whose thickness may vary because of the deposition procedure. The results of calculations for gallium arsenide crystal at various orientations with this lens are shown in Fig. 9. $V(Z)$ curve for the (001) surface can be directly compared with the measurement results [15], and it is in good agreement. Notice that, the $V(Z)$ curves fail to have a rich structure usually observed with isotropic materials. Nevertheless, the difference between the curves are appreciable when $Z$ is made sufficiently negative, i.e., when the object surface is brought closer to the lens than the focal distance. Figs. 10-12 are similar presentations

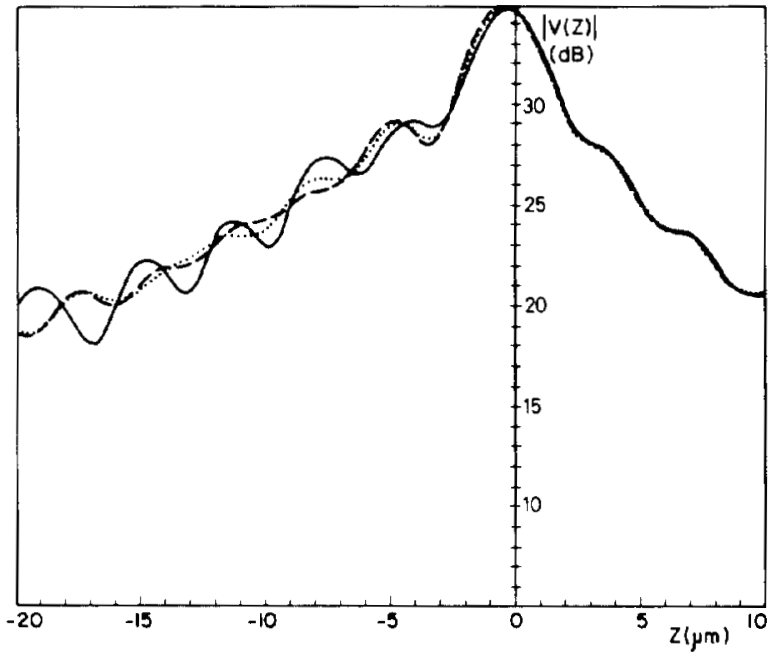

Fig. 10. Calculated $V(Z)$ curves for (001), $(011)$, and (111) faces of nickel shown by solid, dotted, and dashed lines, respectively.

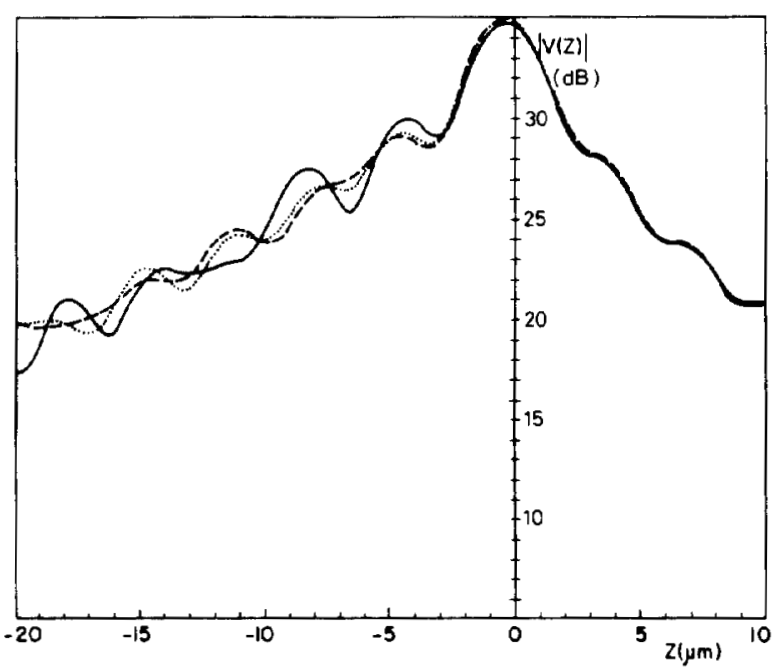

Fig. 11. Calculated $V(Z)$ curves for $(001),(011)$, and (111) faces of iron shown by solid, dotted, and dashed lines, respectively.

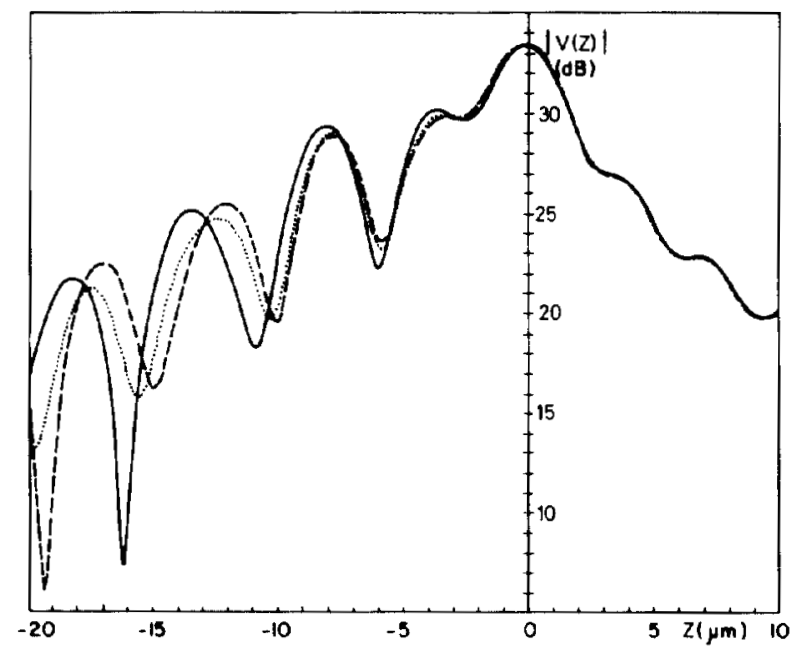

Fig. 12. Calculated $V(Z)$ curves for $(001),(011)$, and (111) faces of aluminum show by solid. dotted, and dashed lines, respectively. 
for nickel, iron and aluminum crystals. The curves there also show that deep nulls and sharp peaks which normally give rise to the high contrast in acoustic images are missing. Apparently, $\phi$ dependent Rayleigh wave velocity washed out the conventional $V(Z)$ effect after the integration operation. To get a contrast between different crystallographic planes of the same material, object must be in an out-of-focus position in the negative $Z$ direction.

The inversion of $V(Z)$ has been used to characterize materials [16]. It is possible to obtain the reflectance function from the measured $V(Z)$ by proper transformations. If this technique is applied to anisotropic materials, the result will be the mean reflectance function defined above.

\section{InCREasing the Sensitivity to Anisotropy}

As mentioned above, the well-known interference mechanism, which is responsible for the material dependence of $V(Z)$ curves, is not particularly successful for anisotropic materials. We must try to find another mechanism to enhance the sensitivity to anisotropy. Inspection of Figs. 1-7 may suggest a way to get an increase in contrast between the surfaces of the same material with different crystallographic orientation. Comparison of the amplitude curves indicate that the reflection coefficients around zero degree do not differ considerably. Use of these reflected rays as a reference is not helpful, since the conventional $V(Z)$ interference mechanism does not work very well. Hence, there is no sense in wasting power for rays near normal incidence. On the other hand, at higher angle values and especially around the phase transition there is more difference. This difference can be emphasized, if power can be concentrated in this region and if only those angles can be excited. Such an insonification can be achieved through an annular type excitation. Nikoonahad et al [21] used an acoustic lens with a suitable transducer to exclude some excitation angles for providing Rayleigh wave suppression. Our proposal here is just the opposite. The central rays are to be suppressed to increase sensitivity to anisotropy. Note that, providing an annular transducer does not guarantee that excitation $u^{+}$ will be annular. Diffraction effects are not at all negligible when it is considered that at typical operating frequencies the wavelength is comparable to the size of the transducer.

\section{A. Annular Excitation}

Achieving an annular insonification is possible easily if the transducer is deposited directly on the curved surface of lens in the desired pattern. For small lenses this may create technological problems. In this case, the annular transducer must be deposited on the opposite and flat surface of the buffer rod at a distance from the lens (Fig. 13). For such a geometry the diffraction loss may be quite high if no optimization is made. Minimization of diffraction loss is important for maximizing the SNR of the acoustic microscope. For this purpose we refer to (4) and use it for a perfect reflector as the object ( $\left.\Omega^{\prime}=1\right)$. Assuming that

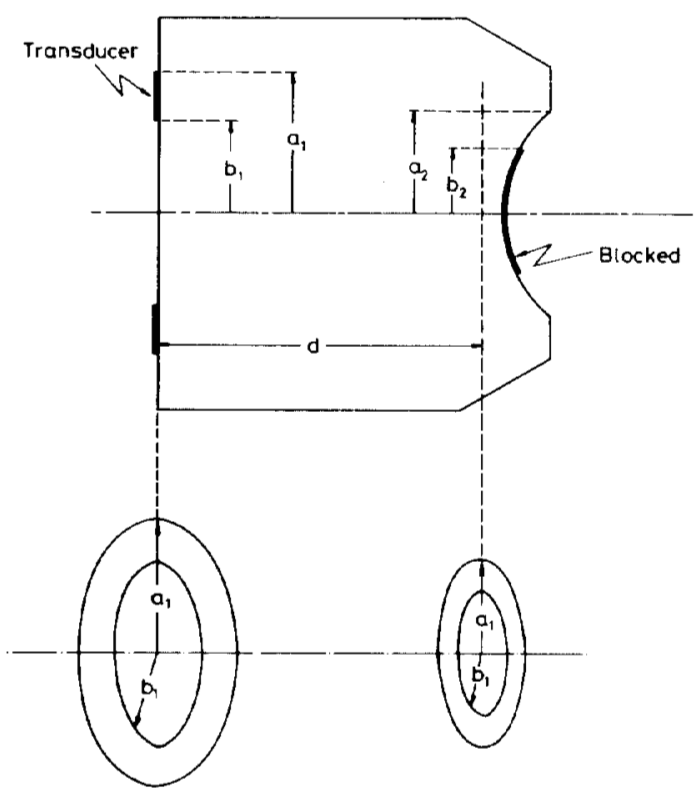

Fig. 13. Geometry used in calculating diffraction loss between two-ring shaped transducers separated by distance.

the functions $u^{+}$and $P$ are purely real (uniform phase), we find the maximum received signal, $V_{\max }$, at $Z=0$ as

$$
\begin{aligned}
V_{\max }= & \left.V(0)\right|_{\mathfrak{R}^{\prime}=1}=K f^{2} \int_{0}^{\sin \theta_{m}} \sin \theta\left[u^{+}(f \sin \theta)\right. \\
& \cdot P(f \sin \theta)]^{2} d(\sin \theta) .
\end{aligned}
$$

Using the Cauchy-Schwartz inequality we can convert the integral on the right to

$$
\begin{aligned}
V_{\max } \geq & \frac{2 K f^{2}}{\sin ^{2} \theta_{m}}\left(\int _ { 0 } ^ { \operatorname { s i n } \theta _ { m } } \operatorname { s i n } \theta \left[u^{+}(f \sin \theta)\right.\right. \\
& \cdot P(f \sin \theta)] d(\sin \theta))^{2}
\end{aligned}
$$

where the equality is satisfied if $u^{+}$and $P$ are constant (uniform insonification). The integral inside the square sign of (5) is proportional to the voltage generated by a fictitious transducer whose shape is defined by the pupil function $P$, when it is insonified by the field $u^{+}$generated by the transducer of the acoustic lens. Hence, minimizing the diffraction loss between these two transducers will help us achieve our goal of maximizing the SNR. The square operation in (5) signifies the two-way propagation of the acoustic waves. Hence, to calculate the diffraction loss encountered in the proposed geometry for annular excitation, we may instead calculate the diffraction loss between the two transducers. Doubling the loss in decibels for such a geometry will approximately give us the required diffraction loss in a pessimistic sense. The approximation involved here gets better, as the insonification function $u^{+}$becomes more uniform that is usually a desired property for a high resolution performance. As depicted in Fig. 13, the ring shaped receiving transducer simulates the lens surface that is suitably blocked for central rays. 
We now turn to calculation of diffraction loss between two ring shaped transducers. An analytical expression for the diffraction loss between two circular transducers was given earlier [22]. The transmission coefficient, $\Gamma$, between two circular longitudinal wave transducers that are located coaxially and facing each other at a distance $d$ can be written to a very good approximation as

$$
\begin{aligned}
\Gamma\left(a_{1}, a_{2}, d\right)= & \frac{4 a_{1} a_{2}}{\pi k_{0}} \int_{0}^{k_{0}} k_{z}^{2} \exp \left(-j k_{z} d\right) \\
& \cdot \operatorname{jinc}\left(\frac{a_{1}}{\pi \sqrt{1-2 b}} \sqrt{k_{0}^{2}-k_{z}^{2}}\right) \\
& \cdot \operatorname{jinc}\left(\frac{a_{2}}{\pi \sqrt{1-2 b}} \sqrt{k_{0}^{2}-k_{z}^{2}}\right) d k_{z}
\end{aligned}
$$

where jinc $(x)=J_{1}(\pi x) / 2 x, J_{1}$ is the first order Bessel function of the first kind, $a_{1}$ and $a_{2}$ are the radii of the two circular transducers, $k_{0}$ is the wavenumber and $b$ is the anisotropy factor in the propagation medium between them. The anisotropy factor is defined by Waterman [23] along or close to pure mode directions and it provides a simple but approximate method of computing diffraction in anisotropic media. The integral in (6) can be easily evaluated when it is converted into a Fourier transform:

$$
\begin{aligned}
\Gamma\left(a_{1}, a_{2}, d\right)= & \frac{4 a_{1} a_{2}}{\pi k_{0}} \mathcal{F}^{-1}\left\{k^{2} \operatorname{jinc}\left(\frac{a_{1}}{\pi} \sqrt{k_{0}^{2}-k^{2}}\right)\right. \\
& \cdot \operatorname{jinc}\left(\frac{a_{2}}{\pi} \sqrt{k_{0}^{2}-k^{2}}\right) \\
& \left.\cdot \operatorname{rect}\left(k / k_{0}-1 / 2\right)\right\}\left.\right|_{x=d} .
\end{aligned}
$$

where $\operatorname{rect}(x)=1$ for $|x| \leq \frac{1}{2}$ and 0 otherwise.

Using this result, one can express the diffraction loss between two ring shaped transducers as follows. Suppose that the two ring transducers have outer radii $a_{1}$ and $a_{2}$, inner radii $b_{1}$ and $b_{2}$, respectively (See Fig. 13). By principle of superposition, the ring transducers can be expressed as the difference of two circular transducers. Taking care of constant factors properly, the transmission coefficient between the two transducers, $\Gamma_{r}$, is

$$
\begin{aligned}
\Gamma_{r}(d)= & \frac{1}{\sqrt{\left(a_{1}^{2}-b_{1}^{2}\right)^{2}\left(a_{2}^{2}-b_{2}^{2}\right)^{2}}}\left\{a_{1} a_{2} \Gamma\left(a_{1}, a_{2}, d\right)\right. \\
& +b_{1} b_{2} \Gamma\left(b_{1}, b_{2}, d\right)-a_{1} b_{2} \Gamma\left(a_{1}, b_{2}, d\right) \\
& \left.-a_{2} b_{1} \Gamma\left(a_{2}, b_{1}, d\right)\right\}
\end{aligned}
$$

We can now relate the maximum received signal to the transmission coefficient as $V_{\max } \propto \Gamma_{r}^{2}(d)$.

First, we kept the size of the transducers the same, i.e. $a_{1}=a_{2}$ and $b_{1}=b_{2}$ and made a number of calculations for different values of $a_{1}$ and $b_{1}$. It was seen that the curves corresponding to different values of $a_{1}$ and $b_{1}$ are barely

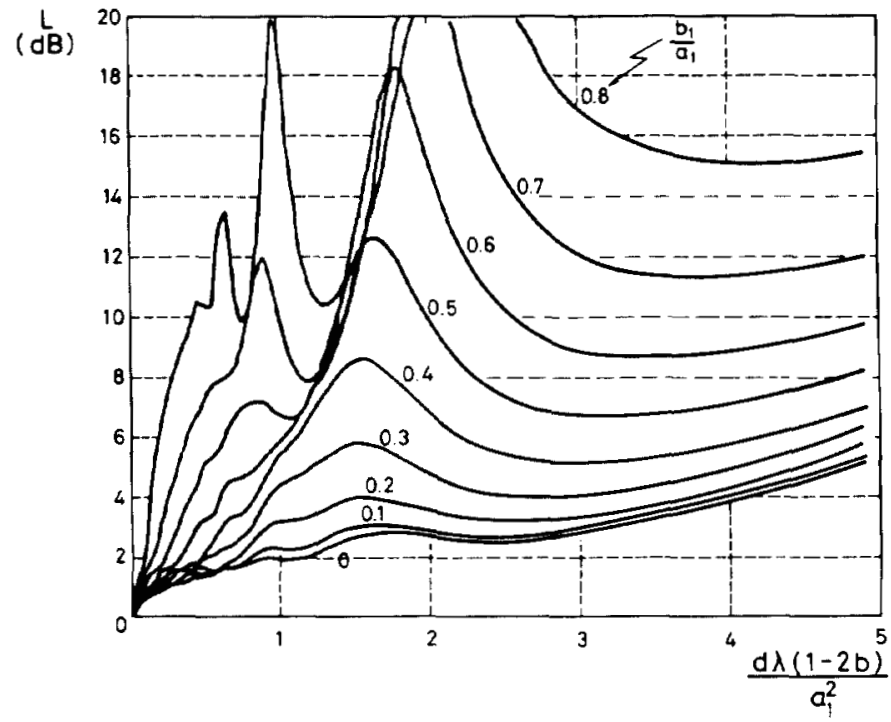

Fig. 14. Diffraction loss between two equal size annular transducers with inner radius $b_{1}$ and outer radius $a_{1}$ as function of separation $d$ between them where $\lambda$ is wavelength in medium and $b$ is anisotropy factor.

distinguishable from each other as long as $b_{1} / a_{1}$ ratio remains the same. This simplifies the presentation of results. One set of curves is applicable to almost all cases. Fig. 14 is a plot of one-way diffraction loss $(L=-20$ $\left.\log \left|\Gamma_{r}\right|\right)$ as a function of distance $(d)$ between the transducers. Inner radius of the annulus is the parameter in the plot. The horizontal axis is normalized with respect to $a_{1}^{2} / \lambda(1-2 b)$, where $\lambda$ is the wavelength in the propagation medium. The vertical axis is normalized such that at $d=0$ the diffraction loss is zero. The curves shown are for $a_{1}=20 \lambda$, but they are nearly independent of the value of $a_{1}$ except when $a_{1}$ is very small. We have used a 16 384-point fast Fourier transform (FFT) in calculating the given curves.

Inspection of Fig. 14 shows that the diffraction loss is quite high in the Fresnel region of the transducer where the acoustic energy tends to collect around the symmetry axis. The minimum diffraction loss occurs at $d \lambda(1-$ $2 b) / a_{3}^{2}=1.1$ for $b_{1} / a_{1}=0.6$, at 1.2 at $b_{1} / a_{1}=0.7$ and at 1.3 for $b_{1} / a_{1}=0.8$, with corresponding diffraction losses of $6.4,8.0$ and $10.2 \mathrm{~dB}$. As the ring gets thinner, the loss increases. Since we have to double this value to find the two-way loss, the only acceptable solution is in the very near field of the transducer. For example, a ring $\left(b_{1} / a_{1}=0.7\right)$ of radius $10 \lambda$ would result in a one-way diffraction loss of less than $2 \mathrm{~dB}$ as long as the rod length is less than $10 \lambda$. This may be ac ${ }^{-}$eptable at low frequencies (10-MHz range) where the wavelength is long, but at high frequencies $(1-\mathrm{GHz}$ range) the resulting buffer rod length is too short to be realizable.

At high frequencies, the near field solution is not practical. We should try to find an acceptable solution in the Fresnel region. Numerical experimentation with various sizes of rings has shown that choosing equal size transducers is not at all-to our surprise-the optimum solution in the Fresnel region. Unequal ring sizes gave lower dif- 
TABLE I

Minimum Diffraction Loss Geometries for Ring-Shaped INSONIFICATION

\begin{tabular}{lllll}
\hline \hline \multicolumn{5}{c}{ Optimum Transducer Size and One-Way Loss } \\
\hline$b_{2} / a_{2}$ & $a_{1} / a_{2}$ & $b_{1} / a_{2}$ & $d \lambda(1-2 b) / a_{2}^{2}$ & Loss (dB) \\
\hline 0.5 & 1.43 & 0.0 & 1.53 & 3.80 \\
0.5 & 0.833 & 0.0 & 2.33 & 4.34 \\
0.6 & 1.43 & 0.0 & 1.63 & 4.69 \\
0.6 & 0.714 & 0.0 & 2.09 & 5.29 \\
0.7 & 1.54 & 0.0 & 1.89 & 6.93 \\
0.7 & 0.588 & 0.0 & 1.80 & 7.76 \\
0.8 & 1.60 & 0.0 & 2.05 & 8.28 \\
0.8 & 0.50 & 0.0 & 1.60 & 10.9 \\
0.9 & 1.66 & 0.0 & 2.22 & 11.33 \\
0.9 & 0.435 & 0.0 & 1.46 & \\
\hline
\end{tabular}

fraction loss values compared to the equal size case. In fact, when one of the transducers is a complete circular transducer, the diffraction loss becomes minimum. Table I summarizes the optimum transducer sizes we found for various $b_{2} / a_{2}$ ratios. Two sets of transducer sizes are given for every $b_{2} / a_{2}$ ratio. One set is for $a_{1}>a_{2}$ and the other is for $a_{1}<a_{2}$. It is seen that a significant improvement in diffraction loss over Fig. 14 is possible with use of these values. Therefore, Fig. 14 should be used as a design aid at low frequencies, and Table I should be consulted at high frequencies.

When the acoustic lens surface is acting as the receiver transducer, the central portions will unavoidably get insonified. To correct the situation, the central portions of the acoustic lens surface may be coated by an absorber material or the antireflection layers may be etched away in this region. We have to note that, a ring shaped insonification will reduce the resolution slightly, because the side lobe level at the focal plane will rise.

\section{B. $V(Z)$ Curves with Annular Excitation}

We will now consider a lens with annular excitation to prove the improvement in sensitivity to anisotropy. To be able to make a comparison we use the same frequency $(1100 \mathrm{MHz}$ ) and the same lens size (cavity radius $105 \mu \mathrm{m}$, pupil radius $75 \mu \mathrm{m}$ ) as above; but we block the central portion of the lens pupil up to the radius $60 \mu \mathrm{m}\left(b_{2} / a_{2}=\right.$ 0.8 ). Using Table $I$, the optimum transducer radius and buffer rod length are found to be $120 \mu \mathrm{m}, 1140 \mu \mathrm{m}$, respectively, resulting in a two-way diffraction loss of about $15 \mathrm{~dB}$, at worst. The insonification function $\left|u^{+}\right|$of (4) for this case is shown in Fig. 8 as dashed lines. Dotted lines in the same figure represent the acoustic field pattern that is blocked. Fig. 15 depicts calculated $V(Z)$ curves for GaAs at various orientations with this annular excitation. Comparing this figure with Fig. 9 we first note a reduction in the signal level that is due to the extra diffraction loss. But more importantly, the difference between the curves corresponding to different orientations increases. Notice also that the difference is significant even at $Z=0$. Hence, grain structure of materials can be observed also at $Z=0$ without losing resolution. A sim-

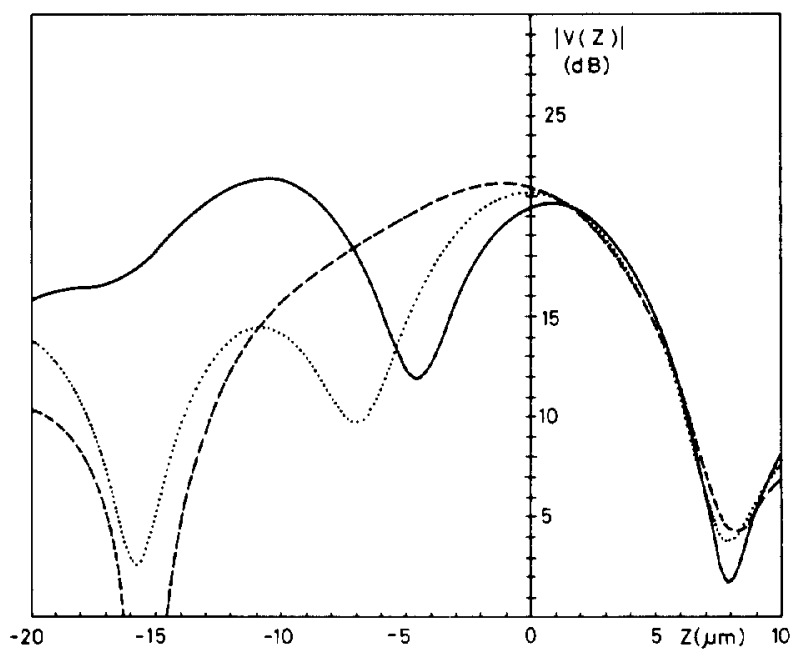

Fig. 15. Calculated $V(Z)$ curves for $(001),(011)$, and (111) faces of gallium arsenide with center blocked lens, shown by solid, dotted, and dashed lines, respectively.

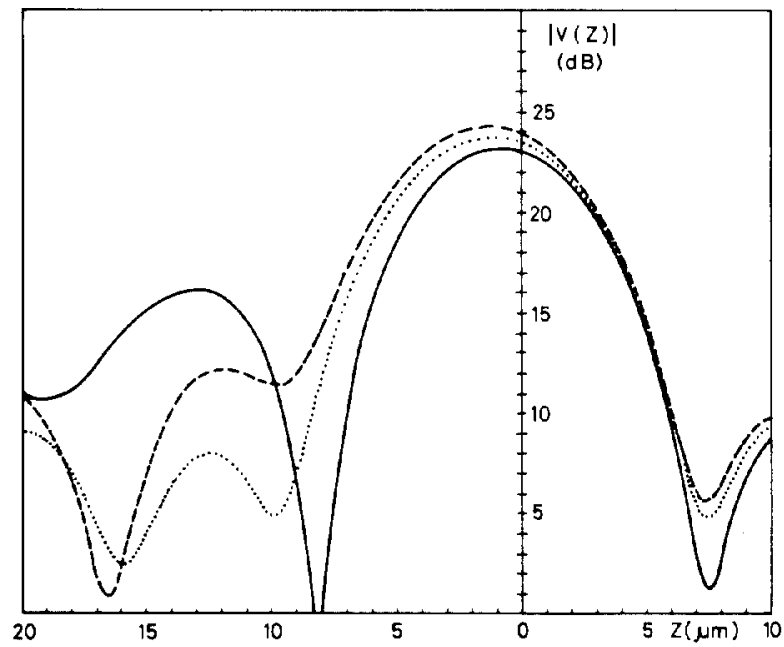

Fig. 16. Calculated $V(Z)$ curves for $(001),(011)$, and (111) faces of nickel, shown by solid, dotted, and dashed lines, respectively, with center blocked lens.

ilar comparison can be made between Figs. 10 and 16 for the case of nickel. Figs. 17 and 18 are corresponding calculation results for iron and aluminum. In all cases, an improvement in sensitivity to anisotropy is obtained, with an SNR sacrifice of about $12 \mathrm{~dB}$. We also point out that the use of ring insonification, not only increased the anisotropy sensitivity, but also the material dependence. For example, iron and nickel are hardly differentiated in Figs. 10 and 11, whereas they are significantly different in Figs. 16 and 17.

It is possible to increase the sensitivity even further, if narrower excitation can be used. But, this is rather difficult because of diffraction effects in the buffer rod. With a very narrow ring type transducer, the buffer rod length should be also very small. Such thin lenses are very difficult to manufacture and they will be very fragile. Moreover, a narrow excitation would reduce the applicability of the lens to different materials. 


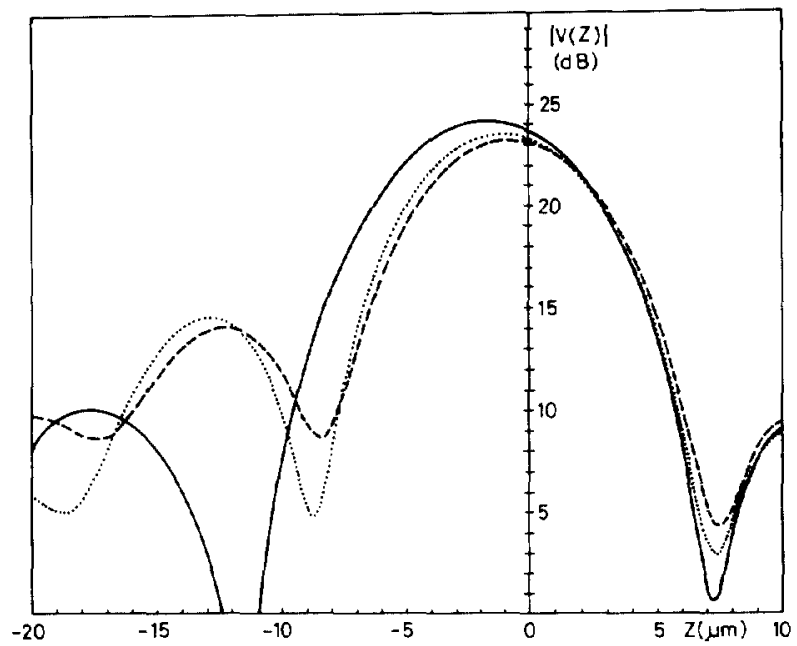

Fig. 17. Calculated $V(Z)$ curves for $(001),(011)$, and $(111)$ faces of iron. shown by solid, dotted, and dashed lines, respectively, with center blocked lens

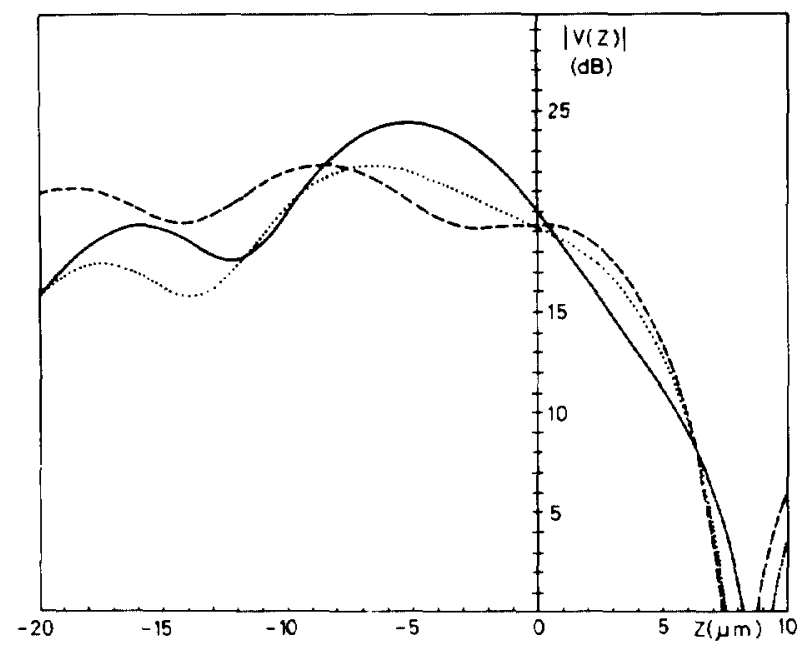

Fig. 18. Calculated $V(Z)$ curves for $(001)$, (011), and (111) faces of alu minum, shown by solid, dotted, and dashed lines, respectively, with center blocked lens.

The approach of Kushibiki et al. gives the highest sensitivity to anisotropy and it is possible to get quantitative information such as surface-wave velocity, but the resulting instrument is not suitable for imaging purposes. The special two-transducer geometry used by Hildebrand and Lam and also by Tan et al. requires special and difficult to manufacture lenses. Since these lenses have separate input and output, they are not directly compatible with the conventional SAMs. The shear wave transducer arrangement used by Khuri-Yakub and Chou has the required characteristics in terms of compatibility with existing systems and high resolution. However, there will be a considerable reflection loss at the lens liquid interface reducing the SNR. Moreover, the spherical aberration in the lens will be higher than the regular lens, because shear waves have lower velocity than the longitudinal waves.

The reflection configuration proposed in this paper to increase sensitivity to anisotropy is relatively simple, di- rectly compatible with existing systems and the loss in resolution is small. But, there is not an easy way to extract quantitative information about object parameters.

\section{Conclusion}

The reflection coefficient of plane acoustic waves at the liquid-solid interface is the most important factor for the material dependent response of the SAM. For an anisotropic solid, the reflection coefficient is a complex function of polar and azimuthal angles of incidence. For circularly symmetric geometries, a mean reflectance function can be defined which is obtained by integrating the reflection coefficient in the azimuthal direction, and thus it depends on polar angle only. The mean reflectance function for most anisotropic materials has a dip around the phase transition angle, because of a cancellation resulting from the direction dependence of the Rayleigh wave velocity. After determination of this function for a given anisotropic material, it is a simple matter to compute the response of an acoustic microscope to that material. We have presented the response of the acoustic microscope to some common anisotropic crystals at their principal planes. The dip of the mean reflectance function near the phase transition reduces the effectiveness of the usual interference mechanism of $V(Z)$ curves. It was seen that a small difference in response exists between different crystallographic surfaces of the same material, provided that the object surface is closer to the lens than the focal length. With such curves, the grain contrast in acoustic images of polycrystalline materials can be predicted.

We have also shown that it is possible to increase the sensitivity of the scanning acoustic microscope to anisotropy by blocking the central portions of the lens cavity. We have presented the calculated response of such an acoustic lens for the same materials to make a comparison. It was seen that the difference in response between different crystalographic planes is enhanced.

We determined the optimal transducer geometry for minimizing the diffraction loss for an annular excitation. At low frequencies, where the lens can be placed in the near field of the transducer, use of a ring shaped transducer gives the lowest diffraction loss. On the other hand, at high frequencies, where the lens can be placed no closer than the Fresnel region, a full circular transducer insonifying a central blocked lens results in the lowest loss. In return for an increased anisotropy sensitivity, some SNR must be sacrificed.

\section{ACKNOWLEDGMENT}

The author would like to thank Orhan Arıkan and Emre Telatar for the development of the reflection coefficient program at the liquid-anisotropic-solid interface.

\section{REFERENCES}

[1] N. J. Burton, "NDT applications of scanning acoustic microscopy," IEE Proc.. vol, 134, no. 3, pt. A. Mar. 1987, pp. 283-289.

[2] J. A Hildebrand. K. Liang, and S. D. Bennett, "Fourier-transform approach to material characterization with the acoustic microscope," J. Appl. Phys, vol. 54, no. 12, pp. 7016-7019, Dec. 1983. 
131 J. Kushibiki, A. Ohkubo, and N. Chubachi, "Anisotropy detection in sapphire by acoustic microscope using line-focus beam," Electron. Lett. vol. 17, pp. 534-536, July 1981.

14] J. Kushibiki and N. Chubachi, "Material characterization by linefocus-beam acoustic microscope," IEEE Trans. Sonics Ulrason., vol. SU-32, no. 2, pp. 189-212, Mar. 1985.

[5] J. A. Hildebrand and L. K. Lam, "Directional acoustic microscopy for observation of elastic anisotropy." Appl. Phys. Lett. . vol. 42, no. 5. pp. 413-415, Mar. 1983.

[6] M. R. T. Tan, H. L. Ransom Jr., C. C. Cutler, and M. Chodorow, "Oblique, off'specular, linear, and nonlinear observations with a scanning micron wavelength acoustic microscope," J. Appl. Phys., vol. 57, no. 11, pp. 4931-4935. June 1985

17] D. A. Davids and H. L. Bertoni, "Bow-tie transducers for measurement of anisotropic materials in acoustic microscopy," in Proc. Ultrason. Symp., Williamsburg, VA, 1986, pp. 735-740.

[8] B. T. Khuri-Yakub and C-H, Chou, "Acoustic microscope lenses with shear wave transducers," in Proc. IEEE Ultrason. Symp. 1986, pp. $741-744$

[9] C-H. Chou, B. T. Khuri-Yakub and K. K. Liang, "Acoustic microscopy with shear wave transducers," in Proc. IEEE Ultrason. Simp., 1987, pp. 813-816.

$[10]$ G. L. Jones and E. G. Henneke II, "Reflection of stress waves at a free boundary in quartz single crystals," IEEE Trans. Sonics Ulurason. vol. SU-20, no. 3, pp. 267-274, July 1973.

111] E. G. Henneke II and G. L. Jones, "Critical angle for reflection at a liquid-solid interface in single crystals," J. Acoust. Soc. Am., vol. 59, no. 1, pp. 204-205, Jan. 1976.

[12] A. Atalar, "Reflection of ultrasonic waves at a liquid-cubic-solid interface," J. Acoust. Soc. Am., vol. 73, no. 2, pp. 435-440. Feb. 1983

[13] M. G. Somekh, G. A. D. Briggs, and C. Ilett, "The effect of elastic anisotropy on contrast in the scanning acoustic microscope," Philosophical Magazine A, vol. 49, no. 2, pp. 179-204, 1984.

114] G. M. Crean and A. Waintal, "Average Rayleigh-wave velocity of a computer simulated erystallographic plane," J. Appl. Crist., vol. 19, pp. 181-187, 1986.

[15] A. Atalar, "An angular spectrum approach to cr ntrast in reflection acoustic microscopy," J. Appl. Phys., vol. 49, no. 10, pp. 51305139. Oct. 1978.

[16] K. K. Liang, G. S. Kino and B. T. Khuri-Yakub, "Material characterization by the inversion of $V(Z)$," IEEE Trans. Sonics Ultrasom., vol. SU-32, no. 2, pp. 213-224. Mar. 1985.

[17] O. Arikan, E. Telatar, and A. Atalar, "Reflection coefficient null of acoustic waves at a liquid-anisotropic-solid interface," J. Acoust. Soc. Am. , Dec. 1988.
[18] B. A. Auld, Acoustic Fields and Waves in Solids, Vols. 1 and 2 New York: Wiley-Interscience, 1973.

[19] M. G. Somekh, private communication.

[20] W. Parmon and H. L. Bertoni. "Ray interpretation of the material signature in the acoustic microscope," Electron. Lett. vol. 15, no. 21. pp. 684-686, Oct. 1979

[21] M. Nikoonahad, P. Sivaprakasapillai, and E. A. Ash. "Rayleigh wave suppression in reflection acoustic microscopy, "Electron. Lett., vol. 19, no. 22, pp. 906-908, Oct. 1983.

[22] A. Atalar, "A fast method of calculating diffraction loss between two facing transducers." IEEE Trans. Ultrason. Ferroelec. Freq. Conir. vol. 35, no. 5, pp. 612-618. Sept. 1988.

[23] P. C. Waterman. "Orientation dependence of elastic waves in single crystals," Phys. Re'l. vol. 113, no. 5. pp. 1240-1253, 1959

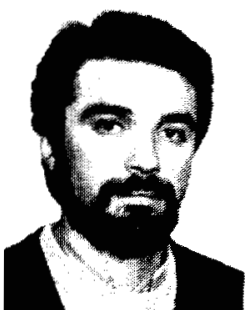

Abdullah Atalar was born in Gaziantep, Turkey, in 1954. He received the B.S. degree in 1974 from Middle East Technical University, Ankara, Turkey, and the M.S. and Ph.D. degrees in 1976 and 1978, respectively, from Stanford University, Stanford, CA, all in electrical engineering. His thesis work was on reflection acoustic microscopy

He was first a Pos1 Doctoral Fellow from 1978 to 1980 and later an Engineering Research Associate at Stanford University, where he continued his work on acoustic microscopy. For eight months he was with Hewlett Packard Labs, Palo Alto, CA, engaged in photoacoustics research. He joined the Middle East Technical University in 1980 as an Assistant Professor. While on leave from the University from 1982 to 1983 , he was with Ernst Leitz Wetzlar, Wetzlar, West Germany, where he was involved in the development of the commercial acoustic microscope. He is presently an Associate Professor and chairman of the Electrical and Electronics En gineering Department at Bilkent University. His current research interests include acoustic imaging, linear acoustics, and computer-aided design in Electrical Engineering.

Dr. Atalar was given the H. Tuğaç Foundation Award of TUBITAK Turkey in 1984 for his contributions to acoustic microscopy. 